\title{
Algebraic relations for reciprocal sums of Fibonacci numbers
}

\author{
by \\ Carsten Elsner (Hannover), Shun Shimomura (Yokohama), \\ and IEkata Shiokawa (Yokohama)
}

1. Introduction. Let $\left\{F_{n}\right\}_{n \geq 0}$ and $\left\{L_{n}\right\}_{n \geq 0}$ be Fibonacci numbers and Lucas numbers defined by

$$
\begin{array}{llll}
F_{0}=0, & F_{1}=1, & F_{n+2}=F_{n+1}+F_{n} & (n \geq 0), \\
L_{0}=2, & L_{1}=1, & L_{n+2}=L_{n+1}+L_{n} & (n \geq 0) .
\end{array}
$$

Duverney, Ke. Nishioka, Ku. Nishioka, and the last named author [9] (see also [8]) proved the transcendence of the numbers

$$
\sum_{n=1}^{\infty} \frac{1}{F_{n}^{2 s}}, \quad \sum_{n=1}^{\infty} \frac{1}{L_{n}^{2 s}}, \quad \sum_{n=1}^{\infty} \frac{1}{F_{2 n-1}^{s}}, \quad \sum_{n=1}^{\infty} \frac{1}{L_{2 n}^{s}} \quad(s=1,2,3, \ldots)
$$

by using Nesterenko's theorem on the Ramanujan functions $P(q), Q(q)$, and $R(q)$ (see Section 3).

In this paper, we prove the algebraic independence of the numbers

$\sum_{n=1}^{\infty} \frac{1}{F_{n}^{2}}, \quad \sum_{n=1}^{\infty} \frac{1}{F_{n}^{4}}, \quad \sum_{n=1}^{\infty} \frac{1}{F_{n}^{6}} \quad\left(\right.$ respectively, $\left.\quad \sum_{n=1}^{\infty} \frac{1}{L_{n}^{2}}, \quad \sum_{n=1}^{\infty} \frac{1}{L_{n}^{4}}, \quad \sum_{n=1}^{\infty} \frac{1}{L_{n}^{6}}\right)$ and write each

$$
\sum_{n=1}^{\infty} \frac{1}{F_{n}^{2 s}} \quad\left(\text { respectively, } \sum_{n=1}^{\infty} \frac{1}{L_{n}^{2 s}}\right) \quad(s=4,5,6, \ldots)
$$

as a rational (respectively, algebraic) function of these three numbers over $\mathbb{Q}$ (see Theorems 1, 3, and Example 1). Similar results are obtained for the alternating sums

$$
\sum_{n=1}^{\infty} \frac{(-1)^{n+1}}{F_{n}^{2 s}} \quad\left(\text { respectively, } \sum_{n=1}^{\infty} \frac{(-1)^{n+1}}{L_{n}^{2 s}}\right) \quad(s=1,2,3, \ldots)
$$

(see Theorems 2 and 4).

2000 Mathematics Subject Classification: Primary 11J81; Secondary 11J91, 33E05.

Key words and phrases: Fibonacci numbers, Lucas numbers. 
It is interesting to compare our results with some arithmetical properties of the values of the Riemann zeta function $\zeta(s)=\sum_{n=1}^{\infty} n^{-s}$ :

(a) Apéry [3] proved the irrationality of $\zeta(3)$ (cf. [4], [6], [15]). It is still unknown whether $\zeta(5)$ is irrational or not. Zudilin [20] showed that at least one of $\zeta(5), \zeta(7), \zeta(9), \zeta(11)$ is irrational.

(b) Euler's formula

$$
\zeta(2 k)=\frac{(-1)^{k-1} 2^{2 k-1} B_{2 k}}{(2 k) !} \pi^{2 k} \quad(k \in \mathbb{N}),
$$

where $B_{2 k}$ are the Bernoulli numbers, implies the algebraic dependence of $\zeta(2 k)$ on $\zeta(2)=\pi^{2} / 6$ for any integer $k \geq 2$.

For Fibonacci numbers we consider the Fibonacci zeta function

$$
\zeta_{\mathrm{F}}(s)=\sum_{n=1}^{\infty} \frac{1}{F_{n}^{s}} \quad(\operatorname{Re}(s)>0),
$$

which extends meromorphically to the whole complex plane (cf. [14]).

$\left(\mathrm{a}^{\prime}\right)$ André-Jeannin [2] proved the irrationality of $\zeta_{\mathrm{F}}(1)$ (see also [5], [7]). The arithmetical nature of $\zeta_{\mathrm{F}}(3)$ is unknown.

$\left(b^{\prime}\right)$ Our results in this paper imply that the values $\zeta_{\mathrm{F}}(2), \zeta_{\mathrm{F}}(4), \zeta_{\mathrm{F}}(6)$ are algebraically independent, and that for any integer $s \geq 4$,

$$
\zeta_{\mathrm{F}}(2 s)-5^{s-2} r_{s} \zeta_{\mathrm{F}}(4) \in \mathbb{Q}(u, v), \quad u:=\zeta_{\mathrm{F}}(2), \quad v:=\zeta_{\mathrm{F}}(6),
$$

with some $r_{s} \in \mathbb{Q}\left(r_{s}=0\right.$ if and only if $s$ is odd $)$, where the rational function of $u$ and $v$ is explicit; for example,

$$
\begin{aligned}
\zeta_{\mathrm{F}}(8)-\frac{15}{14} \zeta_{\mathrm{F}}(4)=\frac{1}{378(4 u+5)^{2}}\left(256 u^{6}-3456 u^{5}+2880 u^{4}+1792 u^{3} v\right. \\
\left.-11100 u^{3}+20160 u^{2} v-10125 u^{2}+7560 u v+3136 v^{2}-1050 v\right)
\end{aligned}
$$

(see Theorem 1, Example 1, and Table 1).

2. Statement of results. Suppose that $\alpha, \beta \in \mathbb{C}$ satisfy $|\beta|<1$ and $\alpha \beta=-1$. We put

$$
\begin{array}{ll}
U_{n}=\frac{\alpha^{n}-\beta^{n}}{\alpha-\beta} & (n \geq 0), \\
V_{n}=\alpha^{n}+\beta^{n} & (n \geq 0) .
\end{array}
$$

If $\alpha+\beta=a \in \mathbb{Z}$, then $\left\{U_{n}\right\}_{n \geq 0}$ and $\left\{V_{n}\right\}_{n \geq 0}$ represent integer-valued, linearly independent binary recurrences satisfying the relation

$$
X_{n+2}=a X_{n+1}+X_{n} \quad(n \geq 0)
$$

with initial values $\left(X_{0}, X_{1}\right)=(0,1)$ and $(2, a)$, respectively. In particular, if $\beta=(1-\sqrt{5}) / 2$, we have the Fibonacci and Lucas numbers:

$$
U_{n}=F_{n}, \quad V_{n}=L_{n} \quad(n \geq 0) .
$$


In what follows $s$ always denotes a positive integer. Set $\sigma_{0}(s)=1$, and for $s \geq 2$ let $\sigma_{1}(s), \ldots, \sigma_{s-1}(s)$ be the elementary symmetric functions of the $s-1$ numbers $-1,-2^{2}, \ldots,-(s-1)^{2}$ defined by

$$
\sigma_{i}(s)=(-1)^{i} \sum_{1 \leq r_{1}<\cdots<r_{i} \leq s-1} r_{1}^{2} \cdots r_{i}^{2} \quad(1 \leq i \leq s-1) .
$$

The coefficients of the expansions

$$
\operatorname{cosec}^{2} x=\frac{1}{x^{2}}+\sum_{j=0}^{\infty} a_{j} x^{2 j}, \quad \sec ^{2} x=\sum_{j=0}^{\infty} b_{j} x^{2 j}
$$

are given by

$$
\begin{aligned}
a_{j-1} & =\frac{(-1)^{j-1}(2 j-1) 2^{2 j} B_{2 j}}{(2 j) !}, \\
b_{j-1} & =\frac{(-1)^{j-1}(2 j-1) 2^{2 j}\left(2^{2 j}-1\right) B_{2 j}}{(2 j) !}
\end{aligned}
$$

$(j \geq 1)$, where $B_{2}=1 / 6, B_{4}=-1 / 30, B_{6}=1 / 42, \ldots$ are the Bernoulli numbers. (In this paper, the symbol $B_{n}$ is used for the Bernoulli numbers only here. It will denote a $q$-series in Sections 3 and 4.)

TheOREm 1. Let $\left\{U_{n}\right\}_{n \geq 1}$ be defined by (1) with $\alpha, \beta \in \overline{\mathbb{Q}}$ satisfying $|\beta|<1$ and $\alpha \beta=-1$, and set

$$
\Phi_{2 s}:=(\alpha-\beta)^{-2 s} \sum_{n=1}^{\infty} \frac{1}{U_{n}^{2 s}} .
$$

Then the numbers $\Phi_{2}, \Phi_{4}, \Phi_{6}$ are algebraically independent, and for any integer $s \geq 4$ the number $\Phi_{2 s}$ is written as

$$
\begin{aligned}
\Phi_{2 s}= & \frac{1}{(2 s-1) !} \\
& \times\left(\sigma_{s-1}(s) \mu_{s}-\sum_{j=1}^{s-1} \frac{(-1)^{j}(2 j) !}{2^{2 j+3}} \sigma_{s-j-1}(s)\left(\varphi_{j}-(-1)^{s} \psi_{j}-a_{j}\right)\right)
\end{aligned}
$$

with

$$
\begin{aligned}
& \mu_{s}= \begin{cases}\Phi_{2} & (\text { s odd }), \\
\frac{1}{3}\left(4 \Phi_{2}^{2}+2 \Phi_{2}-18 \Phi_{4}+\omega-\frac{5}{4}\right) & (\text { s even }),\end{cases} \\
& \varphi_{1}=\frac{4}{3}\left(32 \Phi_{2}^{2}-5 \Phi_{2}-\omega+\frac{13}{10}\right),
\end{aligned}
$$




$$
\begin{aligned}
\varphi_{2} & =-\frac{4}{63}\left(24 \Phi_{2}-1\right)\left(112 \Phi_{2}^{2}-21 \Phi_{2}-5 \omega+\frac{77}{12}\right) \\
\varphi_{j} & =\frac{3}{(j-2)(2 j+3)} \sum_{i=1}^{j-2} \varphi_{i} \varphi_{j-i-1} \quad(j \geq 3) \\
\psi_{1} & =\frac{4}{3}\left(16 \Phi_{2}^{2}-13 \Phi_{2}-5 \omega+\frac{25}{4}\right) \\
\psi_{2} & =\frac{4}{9}\left(24 \Phi_{2}-1\right)\left(16 \Phi_{2}^{2}-13 \Phi_{2}-5 \omega+\frac{25}{4}\right), \\
\psi_{j} & =\frac{1}{j(2 j-1)}\left(2\left(24 \Phi_{2}-1\right) \psi_{j-1}-3 \sum_{i=1}^{j-2} \psi_{i} \psi_{j-i-1}\right) \quad(j \geq 3),
\end{aligned}
$$

where

$$
\omega=\frac{56 \Phi_{6}+5 / 4}{4 \Phi_{2}+1} .
$$

REMARK 1. If $s \geq 4$, then $\left(1+4 \Phi_{2}\right)^{[s / 2]}\left(\Phi_{2 s}-r_{s} \Phi_{4}\right) \in \mathbb{Q}\left[\Phi_{2}, \Phi_{6}\right]$, and the total degree of this does not exceed $s+[s / 2]$, where $r_{s} \in \mathbb{Q}\left(r_{s}=0\right.$ if and only if $s$ is odd).

Table 1. Relations for $\Phi_{2 s}$ in Theorem 1

\begin{aligned} \hline & $x=\Phi_{2} \quad y=\Phi_{4} \quad z=\Phi_{6} \\$\hline$s=4 \quad \Phi_{8}= & \frac{3}{70} y+\frac{1}{1890(4 x+1)^{2}}\left(1280 x^{6}-3456 x^{5}+576 x^{4}+8960 x^{3} z\right. \\ & \left.-444 x^{3}+20160 x^{2} z-81 x^{2}+1512 x z+15680 z^{2}-42 z\right) \\ s=5 \quad \Phi_{10}= & \frac{1}{297(4 x+1)^{2}}\left(512 x^{7}-704 x^{6}+162 x^{5}-1600 x^{4} z-30 x^{4}\right. \\ & \left.+2560 x^{3} z-15 x^{3}+450 x^{2} z+4760 x z^{2}+75 x z+700 z^{2}+15 z\right) \\ s=6 \quad & \frac{1}{462} y+\frac{1}{162162(4 x+1)^{3}}\left(1089536 x^{9}-774144 x^{8}-111168 x^{7}\right. \\ & -4558848 x^{6} z+53424 x^{6}+774144 x^{5} z-40608 x^{5}+1737792 x^{4} z \\ & +8558592 x^{3} z^{2}-11556 x^{4}+311472 x^{3} z+7547904 x^{2} z^{2}-3258 x^{3} \\ & +77112 x^{2} z+1202544 x z^{2}+2458624 z^{3}-351 x^{2}+3780 x z \\ & \left.+127260 z^{2}-252 z\right)\end{aligned}$

Theorem 2. Let $\left\{U_{n}\right\}_{n \geq 1}$ be defined as in Theorem 1 , and set

$$
\Phi_{2 s}^{*}:=(\alpha-\beta)^{-2 s} \sum_{n=1}^{\infty} \frac{(-1)^{n+1}}{U_{n}^{2 s}} .
$$


Then the numbers $\Phi_{2}^{*}, \Phi_{4}^{*}, \Phi_{6}^{*}$ are algebraically independent, and for any integer $s \geq 4$ the number $\Phi_{2 s}^{*}$ is written as

$$
\begin{aligned}
\Phi_{2 s}^{*}= & \frac{1}{(2 s-1) !} \\
& \times\left(\sigma_{s-1}(s) \mu_{s}+\sum_{j=1}^{s-1} \frac{(-1)^{j}(2 j) !}{2^{2 j+3}} \sigma_{s-j-1}(s)\left(\varphi_{j}+(-1)^{s} \psi_{j}-a_{j}\right)\right)
\end{aligned}
$$

with

$$
\begin{aligned}
& \mu_{s}= \begin{cases}\Phi_{2}^{*} & (\text { s odd }), \\
\frac{1}{24}(4 \xi-1) & (\text { s even }),\end{cases} \\
& \varphi_{1}=-\frac{4}{45}\left(180 \Phi_{4}^{*}-10 \xi^{2}+5 \xi-\frac{11}{8}\right), \\
& \varphi_{2}=-\frac{16}{189} \xi\left(180 \Phi_{4}^{*}-6 \xi^{2}+5 \xi-\frac{11}{8}\right), \\
& \varphi_{j}=\frac{3}{(j-2)(2 j+3)} \sum_{i=1}^{j-2} \varphi_{i} \varphi_{j-i-1} \quad(j \geq 3), \\
& \psi_{1}=-\frac{4}{9}\left(180 \Phi_{4}^{*}+2 \xi^{2}+5 \xi-\frac{11}{8}\right), \\
& \psi_{2}=\frac{16}{27} \xi\left(180 \Phi_{4}^{*}+2 \xi^{2}+5 \xi-\frac{11}{8}\right), \\
& \psi_{j}=-\frac{1}{j(2 j-1)}\left(8 \xi \psi_{j-1}+3 \sum_{i=1}^{j-2} \psi_{i} \psi_{j-i-1}\right) \quad(j \geq 3),
\end{aligned}
$$

where $\xi=\xi\left(\Phi_{2}^{*}, \Phi_{4}^{*}, \Phi_{6}^{*}\right)$ is a number satisfying

(3) $8 \xi^{3}+5 \xi^{2}+\left(1440 \Phi_{4}^{*}-46\right) \xi-\left(252 \Phi_{2}^{*}+1260 \Phi_{4}^{*}-7560 \Phi_{6}^{*}-\frac{177}{16}\right)=0$.

Table 2. Relations for $\Phi_{2 s}^{*}$ in Theorem 2

$$
\begin{aligned}
& x=\Phi_{2}^{*} \quad y=\Phi_{4}^{*} \\
s=4 \quad \Phi_{8}^{*}= & \frac{1}{3} y^{2}+\left(\frac{5}{189} \xi^{2}-\frac{11}{378} \xi+\frac{23}{432}\right) y \\
& +\frac{1}{6804} \xi^{4}-\frac{1}{6804} \xi^{3}-\frac{23}{18144} \xi^{2}+\frac{71}{108864} \xi-\frac{143}{1741824} \\
s=5 \quad \Phi_{10}^{*}= & \frac{1}{630} x-\left(\frac{80}{693} \xi-\frac{10}{63}\right) y^{2}-\left(\frac{8}{2079} \xi^{3}-\frac{5}{2079} \xi^{2}+\frac{5}{756} \xi-\frac{5}{756}\right) y \\
& -\frac{1}{56133} \xi^{5}+\frac{1}{64152} \xi^{4}+\frac{403}{4490640} \xi^{3}-\frac{5}{40824} \xi^{2}+\frac{1007}{3265920} \xi-\frac{40739}{574801920}
\end{aligned}
$$


Theorem 3. Let $\left\{V_{n}\right\}_{n \geq 1}$ be defined by (2) with $\alpha, \beta \in \overline{\mathbb{Q}}$ satisfying $|\beta|<1$ and $\alpha \beta=-1$, and set

$$
\Psi_{2 s}:=\sum_{n=1}^{\infty} \frac{1}{V_{n}^{2 s}} .
$$

Then the numbers $\Psi_{2}, \Psi_{4}, \Psi_{6}$ are algebraically independent, and for any integer $s \geq 4$ the number $\Psi_{2 s}$ is written as

$$
\Psi_{2 s}=\frac{1}{(2 s-1) !}\left(\sigma_{s-1}(s) \mu_{s}+\sum_{j=1}^{s-1} \frac{(-1)^{j}(2 j) !}{2^{2 j+3}} \sigma_{s-j-1}(s)\left(\psi_{j}-(-1)^{s}\left(\varphi_{j}-b_{j}\right)\right)\right)
$$

with

$$
\begin{aligned}
& \mu_{s}= \begin{cases}\Psi_{2} & (\text { s odd }), \\
4 \Psi_{2}^{2}+\Psi_{2}-6 \Psi_{4} & (\text { s even }),\end{cases} \\
& \varphi_{1}=\frac{1}{2}\left(8 \Psi_{2}+1\right)\left(8 \Psi_{2}+\eta+1\right), \\
& \varphi_{2}=\frac{1}{12}\left(8 \Psi_{2}+1\right)\left(8 \Psi_{2}+\eta+1\right)\left(24 \Psi_{2}+\eta+3\right), \\
& \varphi_{j}=\frac{1}{j(2 j-1)}\left(\left(24 \Psi_{2}+\eta+3\right) \varphi_{j-1}+3 \sum_{i=1}^{j-2} \varphi_{i} \varphi_{j-i-1}\right) \quad(j \geq 3), \\
& \psi_{1}=-\frac{1}{2}\left(8 \Psi_{2}+1\right)\left(8 \Psi_{2}-\eta+1\right), \\
& \psi_{2}=\frac{1}{12}\left(8 \Psi_{2}+1\right)\left(8 \Psi_{2}-\eta+1\right)\left(24 \Psi_{2}-\eta+3\right), \\
& \psi_{j}=-\frac{1}{j(2 j-1)}\left(\left(24 \Psi_{2}-\eta+3\right) \psi_{j-1}+3 \sum_{i=1}^{j-2} \psi_{i} \psi_{j-i-1}\right) \quad(j \geq 3),
\end{aligned}
$$

Table 3. Relations for $\Psi_{2 s}$ in Theorem 3

$$
\begin{aligned}
& \hline x=\Psi_{2} \quad y=\Psi_{4} \\
& \hline s=4 \quad \Psi_{8}= \frac{3}{70} y+\frac{8}{35} x^{4}+\frac{4}{35} x^{3}+\left(\frac{1}{315} \eta^{2}+\frac{1}{45} \eta+\frac{2}{63}\right) x^{2} \\
&+\left(\frac{1}{1260} \eta^{2}+\frac{1}{180} \eta+\frac{11}{2520}\right) x+\frac{1}{20160} \eta^{2}+\frac{1}{2880} \eta-\frac{1}{2520} \\
& s=5 \quad \Psi_{10}= \frac{2}{15} x^{5}+\frac{1}{12} x^{4}+\left(\frac{17}{5040} \eta^{2}+\frac{11}{504} \eta+\frac{41}{720}\right) x^{3} \\
&+\left(\frac{17}{13440} \eta^{2}+\frac{11}{1344} \eta+\frac{31}{1920}\right) x^{2} \\
&+\left(\frac{1}{5806080} \eta^{4}+\frac{1}{96768} \eta^{3}+\frac{67}{193536} \eta^{2}+\frac{625}{290304} \eta+\frac{2221}{645120}\right) x \\
&+\frac{1}{46448640} \eta^{4}+\frac{1}{774144} \eta^{3}+\frac{233}{7741440} \eta^{2}+\frac{61}{331776} \eta-\frac{1111}{5160960} \\
& \hline
\end{aligned}
$$


where $\eta=\eta\left(\Psi_{2}, \Psi_{6}\right)$ is a number satisfying

$$
(\eta+5)^{2}=-192 \Psi_{2}^{2}-48 \Psi_{2}+6+\frac{3840 \Psi_{6}+30}{8 \Psi_{2}+1} .
$$

Theorem 4. Let $\left\{V_{n}\right\}_{n \geq 1}$ be defined as in Theorem 3 , and set

$$
\Psi_{2 s}^{*}:=\sum_{n=1}^{\infty} \frac{(-1)^{n+1}}{V_{n}^{2 s}} .
$$

Then the numbers $\Psi_{2}^{*}, \Psi_{4}^{*}, \Psi_{6}^{*}$ are algebraically independent, and for any integer $s \geq 4$ the number $\Psi_{2 s}^{*}$ is written as

$\Psi_{2 s}^{*}=\frac{1}{(2 s-1) !}\left(\sigma_{s-1}(s) \mu_{s}+\sum_{j=1}^{s-1} \frac{(-1)^{j}(2 j) !}{2^{2 j+3}} \sigma_{s-j-1}(s)\left(\psi_{j}+(-1)^{s}\left(\varphi_{j}-b_{j}\right)\right)\right)$

with

$\mu_{s}= \begin{cases}\Psi_{2}^{*} & (\text { s odd }), \\ \frac{1}{8}(\theta-1) & (\text { s even }),\end{cases}$

$\varphi_{1}=-\frac{1}{2}\left(96 \Psi_{4}^{*}-\theta^{2}+2 \theta-3\right)$,

$\varphi_{2}=\frac{1}{12 \theta}\left(96 \Psi_{4}^{*}-\theta^{2}+2 \theta-3\right)\left(96 \Psi_{4}^{*}-3 \theta^{2}+2 \theta-3\right)$,

$\varphi_{j}=-\frac{1}{j(2 j-1)}\left(\left(96 \Psi_{4}^{*}-3 \theta^{2}+2 \theta-3\right) \frac{\varphi_{j-1}}{\theta}-3 \sum_{i=1}^{j-2} \varphi_{i} \varphi_{j-i-1}\right) \quad(j \geq 3)$,

$\psi_{1}=-\frac{1}{2}\left(96 \Psi_{4}^{*}+\theta^{2}+2 \theta-3\right)$,

$\psi_{2}=\frac{1}{12 \theta}\left(96 \Psi_{4}^{*}+\theta^{2}+2 \theta-3\right)\left(96 \Psi_{4}^{*}+3 \theta^{2}+2 \theta-3\right)$,

$\psi_{j}=-\frac{1}{j(2 j-1)}\left(\left(96 \Psi_{4}^{*}+3 \theta^{2}+2 \theta-3\right) \frac{\psi_{j-1}}{\theta}+3 \sum_{i=1}^{j-2} \psi_{i} \psi_{j-i-1}\right) \quad(j \geq 3)$,

Table 4. Relations for $\Psi_{2 s}^{*}$ in Theorem 4

$\begin{aligned} & x=\Psi_{2}^{*} \quad y=\Psi_{4}^{*} \\ s=4 \quad \Psi_{8}^{*}= & \frac{1}{35840 \theta^{2}}\left(98304 y^{3}-1024(22 \theta+9) y^{2}\right. \\ & \left.+32\left(11 \theta^{4}+32 \theta^{2}+44 \theta+9\right) y-2 \theta^{5}-11 \theta^{4}+38 \theta^{2}-22 \theta-3\right) \\ s=5 \quad \Psi_{10}^{*}= & \frac{1}{630} x+\frac{1}{645120 \theta}\left(786432 y^{3}-73728(\theta+1) y^{2}\right. \\ & \left.+576\left(\theta^{4}+3 \theta^{2}+8 \theta+4\right) y-3 \theta^{5}-18 \theta^{4}-16 \theta^{3}-54 \theta^{2}+115 \theta-24\right)\end{aligned}$


where $\theta=\theta\left(\Psi_{2}^{*}, \Psi_{4}^{*}, \Psi_{6}^{*}\right)$ is a number satisfying

$$
\theta^{2}-\left(192 \Psi_{4}^{*}-6\right) \theta+1920 \Psi_{6}^{*}-64 \Psi_{2}^{*}-7=0 .
$$

Remark 2. The quantities $\xi, \eta$, and $\theta$ in Theorems 2,3 , and 4 , respectively, are algebraic functions of the corresponding sums for $1 \leq s \leq 3$. In applying these theorems, we have to choose appropriate branches of them depending on the parameter $a$ (or $\beta$ ). This will be done in Section 5 (Theorems 5,6 , and 7 ).

EXAMPLE 1. The reciprocal sums of Fibonacci (respectively, Lucas) numbers $\sum_{n=1}^{\infty} F_{n}^{-2}, \sum_{n=1}^{\infty} F_{n}^{-4}, \sum_{n=1}^{\infty} F_{n}^{-6}$ (respectively, $\sum_{n=1}^{\infty} L_{n}^{-2}, \sum_{n=1}^{\infty} L_{n}^{-4}$, $\sum_{n=1}^{\infty} L_{n}^{-6}$ ) are algebraically independent, and for any integer $s \geq 4$ the number $5^{-s} \sum_{n=1}^{\infty} F_{n}^{-2 s}$ (respectively, $\sum_{n=1}^{\infty} L_{n}^{-2 s}$ ) is written by the formula in Theorem 1 (respectively, Theorem 3 with $\eta$ in Theorem 6). The alternating sums $\sum_{n=1}^{\infty}(-1)^{n+1} F_{n}^{-2 s}$ and $\sum_{n=1}^{\infty}(-1)^{n+1} L_{n}^{-2 s}$ have similar properties mentioned in Theorems 2 and 4 with $\xi$ and $\theta$ in Theorems 5 and 7 , respectively.

Example 2. The Pell numbers $1,2,5,12,29, \ldots$ defined by

$$
P_{0}=0, \quad P_{1}=1, \quad P_{n+2}=2 P_{n+1}+P_{n} \quad(n \geq 0)
$$

are expressible by (1) with $\beta=1-\sqrt{2}$ and $\alpha=-1 / \beta$ satisfying $\alpha-\beta=\sqrt{8}$ (see [13]). To the numbers $\sum_{n=1}^{\infty} P_{n}^{-2 s}$ and $\sum_{n=1}^{\infty}(-1)^{n+1} P_{n}^{-2 s}$, we can apply Theorems 1 and 2 (with Theorem 5).

3. Preparation for the proofs. The reciprocal sums of $\left\{U_{n}\right\}_{n \geq 1}$ and $\left\{V_{n}\right\}_{n \geq 1}$ in our theorems are written as series of hyperbolic functions. In [19] Zucker gave a method of summing such series. He wrote them as $q$-series expressible in closed form in terms of $K, E$, and $k$, where $K$ and $E$ are the complete elliptic integrals of the first and second kind with the modulus $k \neq 0, \pm 1$ defined by

$$
K=K(k)=\int_{0}^{1} \frac{d t}{\sqrt{\left(1-t^{2}\right)\left(1-k^{2} t^{2}\right)}}, \quad E=\int_{0}^{1} \sqrt{\frac{1-k^{2} t^{2}}{1-t^{2}}} d t
$$

for $k^{2} \in \mathbb{C} \backslash(\{0\} \cup\{z \mid z \geq 1\})$. The branch of each integrand is chosen so that it tends to 1 as $t \rightarrow 0$. The relation among $q$ and these quantities is as follows:

$$
q=e^{-\pi c}, \quad c=K^{\prime} / K, \quad K^{\prime}=K\left(k^{\prime}\right), \quad k^{2}+\left(k^{\prime}\right)^{2}=1 .
$$

We start with the following formulas. We choose $c=c(\beta)$ (or $q=q(\beta)$ ) so that $q=e^{-\pi c}=\beta^{2}, \beta=-e^{-\pi c / 2}$. Then by [19, Tables 1(i), 1(ii), 1(iv), 1(iii)], 
(4) $\Sigma_{1}:=2^{-2 s} \sum_{\nu=1}^{\infty} \operatorname{cosech}^{2 s}(\nu \pi c)=\frac{1}{(2 s-1) !} \sum_{j=0}^{s-1} \sigma_{s-j-1}(s) A_{2 j+1}\left(\beta^{2}\right)$,

(5) $\Sigma_{2}:=2^{-2 s} \sum_{\nu=1}^{\infty} \operatorname{sech}^{2 s}(\nu \pi c)=\frac{(-1)^{s-1}}{(2 s-1) !} \sum_{j=0}^{s-1} \sigma_{s-j-1}(s) B_{2 j+1}\left(\beta^{2}\right)$,

(6) $\Sigma_{3}:=2^{-2 s} \sum_{\nu=1}^{\infty} \operatorname{sech}^{2 s} \frac{(2 \nu-1) \pi c}{2}=\frac{(-1)^{s-1}}{(2 s-1) !} \sum_{j=0}^{s-1} \sigma_{s-j-1}(s) D_{2 j+1}\left(\beta^{2}\right)$,

(7) $\Sigma_{4}:=2^{-2 s} \sum_{\nu=1}^{\infty} \operatorname{cosech}^{2 s} \frac{(2 \nu-1) \pi c}{2}=\frac{1}{(2 s-1) !} \sum_{j=0}^{s-1} \sigma_{s-j-1}(s) C_{2 j+1}\left(\beta^{2}\right)$

(note that $\sigma_{i}(s)$ denotes $\alpha_{i}(s)$ in [19]), where

$$
\begin{array}{ll}
A_{2 j+1}(q)=\sum_{n=1}^{\infty} \frac{n^{2 j+1} q^{2 n}}{1-q^{2 n}}, & B_{2 j+1}(q)=\sum_{n=1}^{\infty} \frac{(-1)^{n+1} n^{2 j+1} q^{2 n}}{1-q^{2 n}}, \\
C_{2 j+1}(q)=\sum_{n=1}^{\infty} \frac{n^{2 j+1} q^{n}}{1-q^{2 n}}, & D_{2 j+1}(q)=\sum_{n=1}^{\infty} \frac{(-1)^{n+1} n^{2 j+1} q^{n}}{1-q^{2 n}} .
\end{array}
$$

Our reciprocal sums are expressible by these series of hyperbolic functions:

$$
\begin{aligned}
& \Phi_{2 s}=(\alpha-\beta)^{-2 s}\left(\sum_{\nu=1}^{\infty} \frac{1}{U_{2 \nu-1}^{2 s}}+\sum_{\nu=1}^{\infty} \frac{1}{U_{2 \nu}^{2 s}}\right)=\Sigma_{3}+\Sigma_{1}, \\
& \Psi_{2 s}=\sum_{\nu=1}^{\infty} \frac{1}{V_{2 \nu-1}^{2 s}}+\sum_{\nu=1}^{\infty} \frac{1}{V_{2 \nu}^{2 s}}=\Sigma_{4}+\Sigma_{2}, \\
& \Phi_{2 s}^{*}=(\alpha-\beta)^{-2 s}\left(\sum_{\nu=1}^{\infty} \frac{1}{U_{2 \nu-1}^{2 s}}-\sum_{\nu=1}^{\infty} \frac{1}{U_{2 \nu}^{2 s}}\right)=\Sigma_{3}-\Sigma_{1}, \\
& \Psi_{2 s}^{*}=\sum_{\nu=1}^{\infty} \frac{1}{V_{2 \nu-1}^{2 s}}-\sum_{\nu=1}^{\infty} \frac{1}{V_{2 \nu}^{2 s}}=\Sigma_{4}-\Sigma_{2} .
\end{aligned}
$$

The $q$-series $A_{2 j+1}, B_{2 j+1}, C_{2 j+1}$, and $D_{2 j+1}$ are generated from Fourier expansions of the squares of Jacobian elliptic functions $\mathrm{ns}^{2} z, \mathrm{nc}^{2} z, \mathrm{dn}^{2} z$, and $\mathrm{nd}^{2} z$, respectively, where

$$
\text { ns } z=\frac{1}{\operatorname{sn} z}, \quad \text { nc } z=\frac{1}{\sqrt{1-\operatorname{sn}^{2} z}}, \quad \operatorname{dn} z=\sqrt{1-k^{2} \operatorname{sn}^{2} z}, \quad \text { nd } z=\frac{1}{\operatorname{dn} z},
$$

with $w=\operatorname{sn} z$ defined by

$$
z=\int_{0}^{w} \frac{d w}{\sqrt{\left(1-w^{2}\right)\left(1-k^{2} w^{2}\right)}} .
$$

The power series expansions of these elliptic functions give the expressions 
of the corresponding $q$-series in terms of $K, E$, and $k$ (cf. [10]). For example, we find in [17] the expressions

$$
\left\{\begin{array}{l}
P\left(q^{2}\right):=1-24 A_{1}(q)=\left(\frac{2 K}{\pi}\right)^{2}\left(\frac{3 E}{K}-2+k^{2}\right) \\
Q\left(q^{2}\right):=1+240 A_{3}(q)=\left(\frac{2 K}{\pi}\right)^{4}\left(1-k^{2}+k^{4}\right) \\
R\left(q^{2}\right):=1-504 A_{5}(q)=\left(\frac{2 K}{\pi}\right)^{6} \frac{1}{2}\left(1+k^{2}\right)\left(1-2 k^{2}\right)\left(2-k^{2}\right)
\end{array}\right.
$$

with $q=e^{-\pi c}, c=K^{\prime} / K$.

We recall here the theorem of Nesterenko and its corollary ([16]).

Nesterenko's Theorem. If $\varrho \in \mathbb{C}$ with $0<|\varrho|<1$, then

$$
\text { trans.deg } \operatorname{deg}_{\mathbb{Q}} \mathbb{Q}(\varrho, P(\varrho), Q(\varrho), R(\varrho)) \geq 3 .
$$

Corollary. If $\varrho \in \overline{\mathbb{Q}}$ with $0<|\varrho|<1$, then $P(\varrho), Q(\varrho)$, and $R(\varrho)$ are algebraically independent.

The corollary with (12) implies the following:

LEMmA 1. If $q=e^{-\pi c} \in \overline{\mathbb{Q}}$ with $0<|q|<1$, then $K / \pi, E / \pi$, and $k$ are algebraically independent.

To get the formulas stated in the theorems, we use the recurrence relations satisfied by the coefficients of the power series expansions of Jacobian elliptic functions, which are given by the following lemmas.

LEMMA 2. The coefficients of the expansion

$$
\mathrm{ns}^{2} z=\frac{1}{z^{2}}+\sum_{j=0}^{\infty} c_{j} z^{2 j}
$$

are given by

$$
\begin{gathered}
c_{0}=\frac{1}{3}\left(1+k^{2}\right), c_{1}=\frac{1}{15}\left(1-k^{2}+k^{4}\right), c_{2}=\frac{1}{189}\left(1+k^{2}\right)\left(1-2 k^{2}\right)\left(2-k^{2}\right), \\
(j-2)(2 j+3) c_{j}=3 \sum_{i=1}^{j-2} c_{i} c_{j-i-1} \quad(j \geq 3) .
\end{gathered}
$$

Proof. Since $w=\operatorname{sn} z$ is a solution of $\left(w^{\prime}\right)^{2}=\left(1-w^{2}\right)\left(1-k^{2} w^{2}\right)$ such that $w(0)=0$, the function $u=\mathrm{ns}^{2} z=w^{-2}$ satisfies $\left(u^{\prime}\right)^{2}=4 w^{-6}\left(w^{\prime}\right)^{2}=$ $4 w^{-2}\left(w^{-2}-1\right)\left(w^{-2}-k^{2}\right)$, that is,

$$
\left(u^{\prime}\right)^{2}=4 u(u-1)\left(u-k^{2}\right)
$$

and differentiation of (13) leads us to

$$
u^{\prime \prime}=6 u^{2}-4\left(1+k^{2}\right) u+2 k^{2} .
$$


Substituting $u=z^{-2}+v, v=\sum_{j=0}^{\infty} c_{j} z^{2 j}$ into (14), we have

$$
\begin{aligned}
6 z^{-4}+ & \sum_{j=0}^{\infty} 2 j(2 j-1) c_{j} z^{2 j-2}=6\left(\sum_{j=0}^{\infty} c_{j} z^{2 j}\right)^{2} \\
& +12 z^{-2} \sum_{j=0}^{\infty} c_{j} z^{2 j}-4\left(1+k^{2}\right) \sum_{j=0}^{\infty} c_{j} z^{2 j}+6 z^{-4}-4\left(1+k^{2}\right) z^{-2}+2 k^{2} .
\end{aligned}
$$

Comparing the coefficients of $z^{-2}$ and the constant terms on both sides, we obtain $c_{0}=\left(1+k^{2}\right) / 3$ and $c_{1}=\left(1-k^{2}+k^{4}\right) / 15$. For $j \geq 2$, the coefficients of $z^{2 j-2}$ on both sides satisfy

$$
2 j(2 j-1) c_{j}=6 \sum_{i=0}^{j-1} c_{i} c_{j-i-1}+12 c_{j}-4\left(1+k^{2}\right) c_{j-1} .
$$

Since $1+k^{2}=3 c_{0}$, this can be written in the form

$$
(j-2)(2 j+3) c_{j}=3 \sum_{i=0}^{j-1} c_{i} c_{j-i-1}-6 c_{0} c_{j-1}=3 \sum_{i=1}^{j-2} c_{i} c_{j-i-1} .
$$

When $j=2$, however, both sides of (15) vanish, and $c_{2}$ is not determined uniquely. To compute $c_{2}$, substitute $u=z^{-2}+c_{0}+c_{1} z^{2}+c_{2} z^{4}+\cdots$ into equation (13), and compare the constant terms. Then we have $c_{2}=$ $\left(1+k^{2}\right)\left(1-2 k^{2}\right)\left(2-k^{2}\right) / 189$. Once $c_{0}, c_{1}$, and $c_{2}$ are known, the coefficients $c_{j}(j \geq 3)$ are uniquely determined by $(15)$.

It is also possible to derive the recurrence formula from the proof of Satz 3 in [11, pp. 169-170] concerning the $\wp$-function by choosing $\mathrm{ns}^{2} z=\wp(z)+$ $\left(1+k^{2}\right) / 3, g_{2}=(4 / 3)\left(1-k^{2}+k^{4}\right)$, and $g_{3}=(4 / 27)\left(1+k^{2}\right)\left(1-2 k^{2}\right)\left(2-k^{2}\right)$.

Lemma 3. The coefficients of the expansion

$$
\left(1-k^{2}\right)\left(\mathrm{nc}^{2} z-1\right)=\sum_{j=1}^{\infty} c_{j} z^{2 j}
$$

are given by

$$
\begin{gathered}
c_{1}=1-k^{2}, \quad c_{2}=\frac{1}{3}\left(1-k^{2}\right)\left(2-k^{2}\right), \\
j(2 j-1) c_{1} c_{j}=6 c_{2} c_{j-1}+3 c_{1} \sum_{i=1}^{j-2} c_{i} c_{j-i-1} \quad(j \geq 3) .
\end{gathered}
$$

Proof. Since $\mathrm{nc}^{2} z=\left(1-\mathrm{sn}^{2} z\right)^{-1}$, the function $u=\left(1-k^{2}\right)\left(\mathrm{nc}^{2} z-1\right)$ is a solution of $\left(u^{\prime}\right)^{2}=4 u(u+1)\left(u+1-k^{2}\right)$ such that $u(0)=u^{\prime}(0)=0$, and hence

$$
u^{\prime \prime}=6 u^{2}+4\left(2-k^{2}\right) u+2\left(1-k^{2}\right) .
$$


Substitution of $u=\sum_{j=1}^{\infty} c_{j} z^{2 j}$ yields the equality

$$
\sum_{j=1}^{\infty} 2 j(2 j-1) c_{j} z^{2 j-2}=6\left(\sum_{j=1}^{\infty} c_{j} z^{2 j}\right)^{2}+4\left(2-k^{2}\right) \sum_{j=1}^{\infty} c_{j} z^{2 j}+2\left(1-k^{2}\right) .
$$

Looking at the constant terms, we have $c_{1}=1-k^{2}$. Furthermore, for $j \geq 2$,

$$
2 j(2 j-1) c_{j}=4\left(2-k^{2}\right) c_{j-1}+6 \sum_{i=1}^{j-2} c_{i} c_{j-i-1}
$$

in particular, $c_{2}=\left(2-k^{2}\right) c_{1} / 3=\left(1-k^{2}\right)\left(2-k^{2}\right) / 3$. Multiplying both sides of (16) by $c_{1}$, we obtain the desired recursive relation for $j \geq 3$.

LEMMA 4. The coefficients of the expansion

$$
\left(1-k^{2}\right) \mathrm{nd}^{2} z=1-k^{2}+\sum_{j=1}^{\infty} c_{j} z^{2 j}
$$

are given by

$$
\begin{gathered}
c_{1}=k^{2}\left(1-k^{2}\right), \quad c_{2}=-\frac{1}{3} k^{2}\left(1-k^{2}\right)\left(1-2 k^{2}\right), \\
j(2 j-1) c_{1} c_{j}=6 c_{2} c_{j-1}-3 c_{1} \sum_{i=1}^{j-2} c_{i} c_{j-i-1} \quad(j \geq 3) .
\end{gathered}
$$

Proof. The function $u=\left(1-k^{2}\right) \mathrm{nd}^{2} z=\left(1-k^{2}\right)\left(1-k^{2} \mathrm{sn}^{2} z\right)^{-1}$, which satisfies $\left(u^{\prime}\right)^{2}=4 u(1-u)\left(u-\left(1-k^{2}\right)\right)$, is a solution of

$$
u^{\prime \prime}=-6 u^{2}+4\left(2-k^{2}\right) u-2\left(1-k^{2}\right)
$$

with the initial condition $u(0)=1-k^{2}, u^{\prime}(0)=0$. Substitution of $u=$ $1-k^{2}+v, v=\sum_{j=1}^{\infty} c_{j} z^{2 j}$ yields $c_{1}=k^{2}\left(1-k^{2}\right)$ and, for $j \geq 2$,

$$
j(2 j-1) c_{j}=-2\left(1-2 k^{2}\right) c_{j-1}-3 \sum_{i=1}^{j-2} c_{i} c_{j-i-1}
$$

Multiplying both sides by $c_{1}$ and observing $c_{2}=-\left(1-2 k^{2}\right) c_{1} / 3$, we obtain the desired formula for $j \geq 3$.

LEMMA 5. The coefficients of the expansion

$$
\mathrm{dn}^{2} z=1+\sum_{j=1}^{\infty} c_{j} z^{2 j}
$$


are given by

$$
\begin{gathered}
c_{1}=-k^{2}, \quad c_{2}=\frac{1}{3} k^{2}\left(1+k^{2}\right), \\
j(2 j-1) c_{1} c_{j}=6 c_{2} c_{j-1}-3 c_{1} \sum_{i=1}^{j-2} c_{i} c_{j-i-1} \quad(j \geq 3) .
\end{gathered}
$$

Proof. By definition, $u=\operatorname{dn}^{2} z$ satisfies $\left(u^{\prime}\right)^{2}=4 u(1-u)\left(u-\left(1-k^{2}\right)\right)$, $u(0)=1$. This lemma is verified by the same argument as in the proof of Lemma 4.

\section{Proofs of the theorems}

4.1. Proof of Theorem 1. It follows from (4), (6), and (8) that

$$
\Phi_{2 s}=\frac{1}{(2 s-1) !}\left(\sigma_{s-1}(s) \mu_{s}+\sum_{j=1}^{s-1} \sigma_{s-j-1}(s)\left(A_{2 j+1}-(-1)^{s} D_{2 j+1}\right)\right)
$$

where $\mu_{s}=\left(A_{1}-(-1)^{s} D_{1}\right)$. In particular

$$
\begin{aligned}
& \Phi_{2}=A_{1}+D_{1}, \quad 6 \Phi_{4}=\left(A_{3}-D_{3}\right)-\left(A_{1}-D_{1}\right), \\
& 120 \Phi_{6}=\left(A_{5}+D_{5}\right)-5\left(A_{3}+D_{3}\right)+4\left(A_{1}+D_{1}\right) .
\end{aligned}
$$

Recall the generating functions of $A_{2 j+1}$ and $D_{2 j+1}$ given by

$$
\begin{gathered}
\left(\frac{2 K}{\pi}\right)^{2} \mathrm{~ns}^{2}\left(\frac{2 K x}{\pi}\right)=\frac{4 K(K-E)}{\pi^{2}}+\operatorname{cosec}^{2} x-8 \sum_{j=0}^{\infty}(-1)^{j} A_{2 j+1} \frac{(2 x)^{2 j}}{(2 j) !}, \\
\left(\frac{2 K}{\pi}\right)^{2}\left(1-k^{2}\right) \mathrm{nd}^{2}\left(\frac{2 K x}{\pi}\right)=\frac{4 K E}{\pi^{2}}-8 \sum_{j=0}^{\infty}(-1)^{j} D_{2 j+1} \frac{(2 x)^{2 j}}{(2 j) !}
\end{gathered}
$$

(cf. [19, Tables 1(i), 1(iv)], [12], [18, p. 535], [1]), which yield

$$
\begin{aligned}
& \varphi_{j}:=\left(\frac{2 K}{\pi}\right)^{2 j+2} c_{j}=a_{j}-(-1)^{j} \frac{2^{2 j+3}}{(2 j) !} A_{2 j+1} \quad(j \geq 1), \\
& \psi_{j}:=\left(\frac{2 K}{\pi}\right)^{2 j+2} c_{j}^{\prime}=(-1)^{j+1} \frac{2^{2 j+3}}{(2 j) !} D_{2 j+1} \quad(j \geq 1) .
\end{aligned}
$$

Here $c_{j}$ and $c_{j}^{\prime}$ are the coefficients given by Lemmas 2 and 4 , respectively. Note that

$$
\left\{\begin{array}{l}
8 D_{1}=\left(\frac{2 K}{\pi}\right)^{2}\left(\frac{E}{K}+k^{2}-1\right) \\
16 D_{3}=\left(\frac{2 K}{\pi}\right)^{4} k^{2}\left(1-k^{2}\right), \\
16 D_{5}=\left(\frac{2 K}{\pi}\right)^{6} k^{2}\left(1-k^{2}\right)\left(1-2 k^{2}\right)
\end{array}\right.
$$


(cf. [19, Table 1(iv)] or (21) combined with Lemma 4). Then, by (12) with $q=\beta^{2}$ and (22),

$$
\begin{aligned}
& A_{1}+D_{1}=\frac{1}{24}\left(1-\left(\frac{2 K}{\pi}\right)^{2}\left(1-2 k^{2}\right)\right), \\
& A_{1}-D_{1}=\frac{1}{24}\left(1-\left(\frac{2 K}{\pi}\right)^{2}\left(\frac{6 E}{K}-5+4 k^{2}\right)\right), \\
& A_{3}+D_{3}=-\frac{1}{240}\left(1-\left(\frac{2 K}{\pi}\right)^{4}\left(1+14 k^{2}-14 k^{4}\right)\right), \\
& A_{3}-D_{3}=-\frac{1}{240}\left(1-\left(\frac{2 K}{\pi}\right)^{4}\left(1-16 k^{2}+16 k^{4}\right)\right), \\
& A_{5}+D_{5}=\frac{1}{504}\left(1-\left(\frac{2 K}{\pi}\right)^{6}\left(1-2 k^{2}\right)\left(1-31 k^{2}+31 k^{4}\right)\right), \\
& A_{5}-D_{5}=\frac{1}{504}\left(1-\left(\frac{2 K}{\pi}\right)^{6}\left(1-2 k^{2}\right)\left(1+32 k^{2}-32 k^{4}\right)\right) .
\end{aligned}
$$

Substituting these quantities into (18) and (19), we have expressions of $\Phi_{2}$, $\Phi_{4}$, and $\Phi_{6}$ in terms of $K, E$, and $k$; and then we put

$$
\begin{aligned}
X & :=24 \Phi_{2}-1=-\left(\frac{2 K}{\pi}\right)^{2}\left(1-2 k^{2}\right) \\
Y:= & 1440 \Phi_{4}+11 \\
= & 10\left(\frac{2 K}{\pi}\right)^{2}\left(\frac{6 E}{K}-5+4 k^{2}\right)+\left(\frac{2 K}{\pi}\right)^{4}\left(1-16 k^{2}+16 k^{4}\right), \\
Z:= & -120960 \Phi_{6}+4032 \Phi_{2}+23 \\
= & 2\left(\frac{2 K}{\pi}\right)^{6}\left(1-2 k^{2}\right)\left(1-31 k^{2}+31 k^{4}\right) \\
& +21\left(\frac{2 K}{\pi}\right)^{4}\left(1+14 k^{2}-14 k^{4}\right) .
\end{aligned}
$$

Let us set

$$
x:=\left(\frac{2 K}{\pi}\right)^{2}, \quad y:=\left(\frac{2 K}{\pi}\right)^{2} \frac{E}{K}, \quad z:=\left(\frac{2 K}{\pi}\right)^{2}\left(1-2 k^{2}\right) .
$$

(Note that the same symbols $x, y$, and $z$ denote different quantities in Section 2.) Then the quantities above are written as follows:

$$
\begin{gathered}
X=-z, \quad Y=10(6 y-3 x-2 z)+4 z^{2}-3 x^{2}, \\
Z=\frac{z}{2}\left(31 z^{2}-27 x^{2}\right)+\frac{21}{2}\left(9 x^{2}-7 z^{2}\right) .
\end{gathered}
$$

Solving these equations, we obtain 


$$
\begin{gathered}
x^{2}=\Omega:=\frac{31 X^{3}+147 X^{2}+2 Z}{27(X+7)}, \\
30(2 y-x)=Y-4 X^{2}-20 X+3 \Omega, \quad z=-X .
\end{gathered}
$$

Hence $x, y, z$ are algebraic over $\mathbb{Q}(X, Y, Z)=\mathbb{Q}\left(\Phi_{2}, \Phi_{4}, \Phi_{6}\right)$, and the algebraic independence of $\Phi_{2}, \Phi_{4}, \Phi_{6}$ follows from Lemma 1 .

Substituting (20) and (21) into (17), we obtain the formula for $\Phi_{2 s}$ stated in the theorem. Observe that

$$
\frac{\Omega}{16}=\frac{124}{3} \Phi_{2}^{2}-\frac{22}{3} \Phi_{2}+\frac{103}{48}-\frac{5}{3} \omega \quad \text { with } \quad \omega=\frac{56 \Phi_{6}+5 / 4}{4 \Phi_{2}+1} .
$$

The expression of $\mu_{s}$ is obtained by writing (23) and (24) in terms of $X$, $Y$, and $\Omega$, namely, $\Phi_{2}, \Phi_{4}$, and $\omega$. Furthermore, (20) and (21) for $j=1,2$ together with (12) and (22) imply

$$
\left\{\begin{array}{l}
\varphi_{1}=\frac{1}{15}\left(\frac{2 K}{\pi}\right)^{4}\left(1-k^{2}+k^{4}\right)=\frac{1}{60}\left(3 x^{2}+z^{2}\right), \\
\varphi_{2}=\frac{1}{189}\left(\frac{2 K}{\pi}\right)^{6}\left(1-2 k^{2}\right)\left(2+k^{2}-k^{4}\right)=\frac{z}{756}\left(9 x^{2}-z^{2}\right), \\
\psi_{1}=\left(\frac{2 K}{\pi}\right)^{4}\left(k^{2}-k^{4}\right)=\frac{1}{4}\left(x^{2}-z^{2}\right), \\
\psi_{2}=-\frac{1}{3}\left(\frac{2 K}{\pi}\right)^{2}\left(1-2 k^{2}\right) \psi_{1}=-\frac{z}{3} \psi_{1}
\end{array}\right.
$$

with $x^{2}=\Omega, z=-X$, from which the desired expressions follow. Multiplying both sides of the recurrence formula in Lemma 2 by $(2 K / \pi)^{2 j+2}$, and using (20), we obtain the formula for $\varphi_{j}(j \geq 3)$. The recurrence formula for $\psi_{j}$ is obtained from Lemma 4 by using $\psi_{2} / \psi_{1}=X / 3=\left(24 \Phi_{2}-1\right) / 3$.

4.2. Proof of Remark 1. Denoting the denominator of $\omega$ by $T:=4 \Phi_{2}+1$, we have the polynomials $T^{\nu} \varphi_{2 \nu-1}, T^{\nu} \varphi_{2 \nu}, T^{\nu} \psi_{2 \nu-1}, T^{\nu} \psi_{2 \nu}(\nu \geq 1)$ in $\left(\Phi_{2}, \Phi_{6}\right)$ of total degrees, say, $\delta_{2 \nu-1}, \delta_{2 \nu}, \delta_{2 \nu-1}^{\prime}, \delta_{2 \nu}^{\prime}$, respectively. Then the recurrence formulas concerning $\varphi_{j}$ and $\psi_{j}$ imply, for $\nu \geq 1$,

$$
\begin{aligned}
\delta_{2 \nu-1} & \leq \max _{1 \leq i \leq 2 \nu-3}\left\{1+\delta_{2 i}+\delta_{2(\nu-i-1)}, \delta_{2 i-1}+\delta_{2(\nu-i)-1}\right\}, \\
\delta_{2 \nu} & \leq \max _{1 \leq i \leq 2 \nu-2}\left\{\delta_{2 i}+\delta_{2(\nu-i)-1}\right\}, \\
\delta_{2 \nu-1}^{\prime} & \leq \max _{1 \leq i \leq 2 \nu-3}\left\{1+\delta_{2 i}^{\prime}+\delta_{2(\nu-i-1)}^{\prime}, \delta_{2 i-1}^{\prime}+\delta_{2(\nu-i)-1}^{\prime}, 2+\delta_{2 \nu-2}^{\prime}\right\}, \\
\delta_{2 \nu}^{\prime} & \leq \max _{1 \leq i \leq 2 \nu-2}\left\{\delta_{2 i}^{\prime}+\delta_{2(\nu-i)-1}^{\prime}, 1+\delta_{2 \nu-1}^{\prime}\right\} .
\end{aligned}
$$

Using these relations, by induction on $\nu$ we can verify $\delta_{2 \nu-1}, \delta_{2 \nu-1}^{\prime} \leq 3 \nu$ and $\delta_{2 \nu}, \delta_{2 \nu}^{\prime} \leq 3 \nu+1$, which yields the estimate for the total degree of the polynomial $\bar{T}^{[s / 2]}\left(\Phi_{2 s}-r_{s} \Phi_{4}\right)$. 
4.3. Proof of Theorem 2. From (4), (6), and (10) we have (32)

$$
\begin{aligned}
\Phi_{2 s}^{*}= & \frac{1}{(2 s-1) !} \\
& \times\left(\sigma_{s-1}(s) \mu_{s}-\sum_{j=1}^{s-1} \sigma_{s-j-1}(s)\left((-1)^{s} D_{2 j+1}\left(\beta^{2}\right)+A_{2 j+1}\left(\beta^{2}\right)\right)\right)
\end{aligned}
$$

with $\mu_{s}=-\left((-1)^{s} D_{1}\left(\beta^{2}\right)+A_{1}\left(\beta^{2}\right)\right)$. In particular,

$$
\begin{aligned}
& \Phi_{2}^{*}=D_{1}-A_{1}, \quad 6 \Phi_{4}^{*}=-\left(D_{3}+A_{3}\right)+\left(D_{1}+A_{1}\right), \\
& 120 \Phi_{6}^{*}=\left(D_{5}-A_{5}\right)-5\left(D_{3}-A_{3}\right)+4\left(D_{1}-A_{1}\right) .
\end{aligned}
$$

Substituting (23)-(26) and (28) into (33) and (34), we obtain

$$
\begin{aligned}
Y & :=1440 \Phi_{4}^{*}-11=-10\left(\frac{2 K}{\pi}\right)^{2}\left(1-2 k^{2}\right)-\left(\frac{2 K}{\pi}\right)^{4}\left(1+14 k^{2}-14 k^{4}\right), \\
Z & :=120960 \Phi_{6}^{*}-4032 \Phi_{2}^{*}+23 \\
& =21\left(\frac{2 K}{\pi}\right)^{4}\left(1-16 k^{2}+16 k^{4}\right)+2\left(\frac{2 K}{\pi}\right)^{6}\left(1-2 k^{2}\right)\left(1+32 k^{2}-32 k^{4}\right),
\end{aligned}
$$

which are written in the form

$$
Y=-10 z-\left(9 x^{2}-7 z^{2}\right) / 2, \quad Z=21\left(4 z^{2}-3 x^{2}\right)+2 z\left(9 x^{2}-8 z^{2}\right)
$$

with $x$ and $z$ given by (30). From these equations, we obtain

$$
8 \xi^{3}+5 \xi^{2}+(Y-35) \xi-(14 Y-Z) / 16=0, \quad \xi=z / 4,
$$

which is written as (3). It is easy to see that $K / \pi, E / \pi$, and $k$ are algebraic functions of $\Phi_{2}^{*}, \Phi_{4}^{*}, \Phi_{6}^{*}$ over $\mathbb{Q}$. This fact combined with Lemma 1 implies the algebraic independence of $\Phi_{2}^{*}, \Phi_{4}^{*}, \Phi_{6}^{*}$.

Formula (32) together with (20) and (21) implies the expression of $\Phi_{2 s}^{*}$ in terms of $\varphi_{j}$ and $\psi_{j}$. By (23) and (24), we can write $\mu_{s}$ in terms of $\Phi_{2}^{*}$ and $\xi$. Substituting $z=4 \xi$ and $9 x^{2}=16\left(7 \xi^{2}-5 \xi\right)-2 Y$ into (31), we obtain $\varphi_{1}, \ldots, \psi_{2}$ expressed in terms of $\Phi_{4}^{*}$ and $\xi$. The recurrence formula for $\psi_{j}$ follows from that of Lemma 4 combined with (21) and the relation $\psi_{2} / \psi_{1}=-4 \xi / 3$.

4.4. Proof of Theorem 3. From (5), (7), and (9) we have

$$
\Psi_{2 s}=\frac{1}{(2 s-1) !}\left(\sigma_{s-1}(s) \mu_{s}+\sum_{j=1}^{s-1} \sigma_{s-j-1}(s)\left(C_{2 j+1}-(-1)^{s} B_{2 j+1}\right)\right),
$$

where $\mu_{s}=\left(C_{1}-(-1)^{s} B_{1}\right)$. In particular,

$$
\begin{aligned}
& \Psi_{2}=C_{1}+B_{1}, \quad 6 \Psi_{4}=\left(C_{3}-B_{3}\right)-\left(C_{1}-B_{1}\right), \\
& 120 \Psi_{6}=\left(C_{5}+B_{5}\right)-5\left(C_{3}+B_{3}\right)+4\left(C_{1}+B_{1}\right) .
\end{aligned}
$$


The formulas

$$
\begin{aligned}
\left(\frac{2 K}{\pi}\right)^{2}\left(1-k^{2}\right)\left(\mathrm{nc}^{2}\left(\frac{2 K x}{\pi}\right)-1\right) & =-\frac{4 K E}{\pi^{2}}+\sec ^{2} x+8 \sum_{j=0}^{\infty}(-1)^{j} B_{2 j+1} \frac{(2 x)^{2 j}}{(2 j) !}, \\
\left(\frac{2 K}{\pi}\right)^{2} \operatorname{dn}^{2}\left(\frac{2 K x}{\pi}\right) & =\frac{4 K E}{\pi^{2}}+8 \sum_{j=0}^{\infty}(-1)^{j} C_{2 j+1} \frac{(2 x)^{2 j}}{(2 j) !}
\end{aligned}
$$

(cf. [19, Tables 1(ii), 1(iii)]) imply the relations

$$
\begin{array}{ll}
\varphi_{j}:=\left(\frac{2 K}{\pi}\right)^{2 j+2} c_{j}=b_{j}+(-1)^{j} \frac{2^{2 j+3}}{(2 j) !} B_{2 j+1} & (j \geq 1), \\
\psi_{j}:=\left(\frac{2 K}{\pi}\right)^{2 j+2} c_{j}^{\prime}=(-1)^{j} \frac{2^{2 j+3}}{(2 j) !} C_{2 j+1} \quad(j \geq 1),
\end{array}
$$

where $c_{j}$ and $c_{j}^{\prime}$ are the coefficients given in Lemmas 3 and 5 , respectively. From [19, Tables 1(ii), 1(iii)] or Lemmas 3 and 5, we obtain

$$
\begin{aligned}
1+8 B_{1} & =\frac{4 K E}{\pi^{2}}, & 8 C_{1} & =\left(\frac{2 K}{\pi}\right)^{2}\left(1-\frac{E}{K}\right), \\
1-16 B_{3} & =\left(\frac{2 K}{\pi}\right)^{4}\left(1-k^{2}\right), & 16 C_{3} & =\left(\frac{2 K}{\pi}\right)^{4} k^{2}, \\
1+8 B_{5} & =\left(\frac{2 K}{\pi}\right)^{6} \frac{1}{2}\left(1-k^{2}\right)\left(2-k^{2}\right), & 16 C_{5} & =\left(\frac{2 K}{\pi}\right)^{6} k^{2}\left(1+k^{2}\right) .
\end{aligned}
$$

Hence

$$
\begin{aligned}
& C_{1}+B_{1}=-\frac{1}{8}+\frac{1}{8}\left(\frac{2 K}{\pi}\right)^{2}, \\
& C_{1}-B_{1}=\frac{1}{8}+\frac{1}{8}\left(\frac{2 K}{\pi}\right)^{2}\left(1-\frac{2 E}{K}\right), \\
& C_{3}+B_{3}=\frac{1}{16}-\frac{1}{16}\left(\frac{2 K}{\pi}\right)^{4}\left(1-2 k^{2}\right), \\
& C_{3}-B_{3}=-\frac{1}{16}+\frac{1}{16}\left(\frac{2 K}{\pi}\right)^{4}, \\
& C_{5}+B_{5}=-\frac{1}{8}+\frac{1}{8}\left(\frac{2 K}{\pi}\right)^{6}\left(1-k^{2}+k^{4}\right), \\
& C_{5}-B_{5}=\frac{1}{8}-\frac{1}{8}\left(\frac{2 K}{\pi}\right)^{6}\left(1-2 k^{2}\right) .
\end{aligned}
$$


So we can express $\Psi_{2}, \Psi_{4}$, and $\Psi_{6}$ in terms of $K, E$, and $k$. Put

$$
\begin{aligned}
X & :=8 \Psi_{2}+1=\left(\frac{2 K}{\pi}\right)^{2}, \\
Y & :=96 \Psi_{4}+3=\left(\frac{2 K}{\pi}\right)^{4}-2\left(\frac{2 K}{\pi}\right)^{2}\left(1-\frac{2 E}{K}\right), \\
Z & :=1920 \Psi_{6}+15 \\
& =2\left(\frac{2 K}{\pi}\right)^{6}\left(1-k^{2}+k^{4}\right)+5\left(\frac{2 K}{\pi}\right)^{4}\left(1-2 k^{2}\right)+8\left(\frac{2 K}{\pi}\right)^{2} .
\end{aligned}
$$

By the same argument as in Sections 4.1 and 4.3 with $x, y, z$ given by (30), we have

$$
\begin{gathered}
x=X, \quad 4 y=Y-X^{2}+2 X, \\
X z^{2}+10 X z+\left(3 X^{3}+16 X-2 Z\right)=0,
\end{gathered}
$$

implying that $x, y$, and $z$ are algebraic over $\mathbb{Q}(X, Y, Z)=\mathbb{Q}\left(\Psi_{2}, \Psi_{4}, \Psi_{6}\right)$.

Substitution of (36) and (37) into (35) yields the desired formula for $\Psi_{2 s}$ in terms of $\varphi_{j}$ and $\psi_{j}$. Furthermore, using (36) and (37) for $j=1,2$ together with Lemmas 3 and 5, respectively, we have

$$
\left\{\begin{array}{l}
\varphi_{1}=\left(\frac{2 K}{\pi}\right)^{4}\left(1-k^{2}\right)=\frac{x}{2}(z+x), \\
\varphi_{2}=\frac{1}{3}\left(\frac{2 K}{\pi}\right)^{2}\left(2-k^{2}\right) \varphi_{1}=\frac{1}{6}(z+3 x) \varphi_{1}, \\
\psi_{1}=-\left(\frac{2 K}{\pi}\right)^{4} k^{2}=\frac{x}{2}(z-x), \\
\psi_{2}=-\frac{1}{3}\left(\frac{2 K}{\pi}\right)^{2}\left(1+k^{2}\right) \psi_{1}=\frac{1}{6}(z-3 x) \psi_{1} .
\end{array}\right.
$$

The right hand sides of these equalities as well as $\mu_{s}$ can be written in terms of $X$ and $z$ satisfying the quadratic equation (45). The recurrence formulas for $\varphi_{j}$ and $\psi_{j}$ are derived from those of Lemmas 3 and 5 combined with $\varphi_{2} / \varphi_{1}=(3 X+z) / 6$ and $\psi_{2} / \psi_{1}=-(3 X-z) / 6$, respectively. Replacing $z$ by $\eta$, we obtain the theorem.

4.5. Proof of Theorem 4. Relation (11) together with (5) and (7) implies

$$
\begin{aligned}
\Psi_{2 s}^{*}= & \frac{1}{(2 s-1) !} \\
& \times\left(\sigma_{s-1}(s) \mu_{s}+\sum_{j=1}^{s-1} \sigma_{s-j-1}(s)\left(C_{2 j+1}\left(\beta^{2}\right)+(-1)^{s} B_{2 j+1}\left(\beta^{2}\right)\right)\right)
\end{aligned}
$$


with $\mu_{s}=C_{1}\left(\beta^{2}\right)+(-1)^{s} B_{1}\left(\beta^{2}\right)$. In particular,

$$
\begin{aligned}
& \Psi_{2}^{*}=C_{1}-B_{1}, \quad 6 \Psi_{4}^{*}=\left(C_{3}+B_{3}\right)-\left(C_{1}+B_{1}\right), \\
& 120 \Psi_{6}^{*}=\left(C_{5}-B_{5}\right)-5\left(C_{3}-B_{3}\right)+4\left(C_{1}-B_{1}\right) .
\end{aligned}
$$

Substituting (38)-(41) and (43) into (48) and (49), we obtain

$$
\begin{aligned}
& Y:=96 \Psi_{4}^{*}-3=-\left(\frac{2 K}{\pi}\right)^{4}\left(1-2 k^{2}\right)-2\left(\frac{2 K}{\pi}\right)^{2}, \\
& Z:=1920 \Psi_{6}^{*}-64 \Psi_{2}^{*}-7=-2\left(\frac{2 K}{\pi}\right)^{6}\left(1-2 k^{2}\right)-5\left(\frac{2 K}{\pi}\right)^{4},
\end{aligned}
$$

which are written as $Y=-x z-2 x, Z=-2 x^{2} z-5 x^{2}$ with $x$ and $z$ given by (30). Then we have $x^{2}-2 Y x+Z=0, z=-2-Y / x$. The quantities $K / \pi, E / \pi$, and $k$ are algebraic functions of $\Psi_{2}^{*}, \Psi_{4}^{*}, \Psi_{6}^{*}$ over $\mathbb{Q}$, which implies the algebraic independence of $\Psi_{2}^{*}, \Psi_{4}^{*}, \Psi_{6}^{*}$.

The desired expression of $\Psi_{2 s}^{*}$ is obtained by substituting (36) and (37) into (47). By (38) and (39) the quantity $\mu_{s}$ is expressible in terms of $\Psi_{2}^{*}$ and $x$. Using (46), we have the desired expressions of $\varphi_{1}, \ldots, \psi_{2}$ in terms of $\Psi_{4}^{*}$ and $x$. Observing the relations $\varphi_{2} / \varphi_{1}=(3 x+z) / 6$ and $\psi_{2} / \psi_{1}=$ $-(3 x-z) / 6$, we derive from Lemmas 3 and 5 the recurrence formulas for $\varphi_{j}$ and $\psi_{j}$, respectively. Replacing $x$ by $\theta$ in these expressions, we obtain the theorem.

5. Branches of the related algebraic functions. In what follows we put $a=\alpha+\beta \in \mathbb{C}$, namely $\beta=(a / 2)\left(1-\sqrt{1+4 a^{-2}}\right)$, where the branch is chosen so that $\beta(a)=O\left(a^{-1}\right)$ as $a \rightarrow \infty$. Then each reciprocal sum in the theorems is treated as a function of $a$ or $\beta$.

THEOREM 5. Under the assumptions of Theorem 2, we have the following:

(i) The function $\xi=\xi(a)$ is holomorphic in the domain $|a|>8.146$, and is expressible in the form

$$
\xi(a)=-\frac{5}{24}+\varrho_{0}+\gamma_{1} / \varrho_{0}, \quad \varrho_{0}=\varrho_{0}(a):=\sqrt[3]{\gamma_{2}+\sqrt{\gamma_{1}^{3}-\gamma_{2}^{2}}} i
$$

with $\gamma_{1}=\gamma_{1}(a), \gamma_{2}=\gamma_{2}(a)$ expressed as

$\gamma_{1}:=\frac{1129}{24^{2}}-60 \Phi_{4}^{*}, \quad \gamma_{2}:=-\frac{17963}{24^{3}}+\frac{63}{4} \Phi_{2}^{*}+\frac{195}{2} \Phi_{4}^{*}-\frac{945}{2} \Phi_{6}^{*}$

satisfying $\gamma_{1}(a)=1129 \cdot 24^{-2}+O\left(a^{-2}\right)$ and $\gamma_{2}(a)=-17963 \cdot 24^{-3}+$ $O\left(a^{-2}\right)$ as $a \rightarrow \infty$. Here the branches of the square and cube roots are taken so that $\sqrt{\gamma_{1}(\infty)^{3}-\gamma_{2}(\infty)^{2}}=(7 / 192) \sqrt{4395}$ and that

$$
\varrho_{0}(\infty)=\frac{1}{48}(11-\sqrt{4395} i) \text {. }
$$


(ii) For any real number $a \geq 2.6$, the function $\xi(a)$ admits the expression (50) with the same branches as above, where

$$
\gamma_{1}^{3}-\gamma_{2}^{2}>0, \quad \gamma_{2}+\sqrt{\gamma_{1}^{3}-\gamma_{2}^{2}} i \neq 0 .
$$

(iii) For $a=1$ (respectively, $a=2$ ) corresponding to the Fibonacci (respectively, Pell) numbers, the quantity $\xi(1)=-2.66158 \ldots$ (respectively, $\xi(2)=-0.72378 \ldots$...) is expressible as (50) with

$$
\varrho_{0}(1)=-1.22662 \ldots+(0.57598 \ldots) i, \gamma_{1}(1)^{3}-\gamma_{2}(1)^{2}>0
$$

(respectively,

$$
\left.\varrho_{0}(2)=-0.25772 \ldots-(1.00662 \ldots) i, \gamma_{1}(2)^{3}-\gamma_{2}(2)^{2}>0\right) .
$$

THEOREM 6. Under the assumptions of Theorem 3, we have the following:

(i) The function $\eta=\eta(a)$ is holomorphic in the domain $|a|>5.431$, and is expressible in the form

$$
\eta(a)=-5+\sqrt{\chi(a)}
$$

with

$$
\chi(a)=-192 \Psi_{2}^{2}-48 \Psi_{2}+6+\left(3840 \Psi_{6}+30\right) /\left(8 \Psi_{2}+1\right)
$$

satisfying $\chi(a)=36+O\left(a^{-2}\right)$ as $a \rightarrow \infty$. Here the branch is taken so that $\sqrt{\chi(\infty)}=6$.

(ii) For $a=1$ corresponding to the Lucas numbers,

$$
\eta(1)=-5-\sqrt{\chi(1)} \quad(<-5),
$$

and for any integer $a \geq 2$

$$
\eta(a)=-5+\sqrt{\chi(a)} \quad(>-5)
$$

Furthermore, the last equality holds for any real number $a \geq 2.4$.

THEOREM 7. Under the assumptions of Theorem 4, we have the following:

(i) The function $\theta=\theta(a)$ is holomorphic in the domain $|a|>5.819$, and is expressible in the form

$$
\theta(a)=\chi_{1}(a)+\sqrt{\chi_{2}(a)}
$$

with

$$
\begin{aligned}
& \chi_{1}(a)=96 \Psi_{4}^{*}-3, \\
& \chi_{2}(a)=64 \Psi_{2}^{*}+9216\left(\Psi_{4}^{*}\right)^{2}-576 \Psi_{4}^{*}-1920 \Psi_{6}^{*}+16
\end{aligned}
$$

satisfying $\chi_{1}(a)=-3+O\left(a^{-4}\right)$ and $\chi_{2}(a)=16+O\left(a^{-2}\right)$ as $a \rightarrow \infty$. Here the branch of the square root is taken so that $\sqrt{\chi_{2}(\infty)}=4$.

(ii) For $a=1$ corresponding to the Lucas numbers,

$$
\theta(1)=\chi_{1}(1)-\sqrt{\chi_{2}(1)} \quad\left(<\chi_{1}(1)\right),
$$


and for any integer $a \geq 2$,

$$
\theta(a)=\chi_{1}(a)+\sqrt{\chi_{2}(a)} \quad\left(>\chi_{1}(a)\right) .
$$

Furthermore, the last equality holds for any real number $a \geq 2.5$.

5.1. Proof of Theorem 6. By definition we have $\left|V_{n}\right|=\left|\beta^{-n}+\beta^{n}\right| \geq$ $|\beta|^{-n}\left(1-|\beta|^{2 n}\right)$, so that $\left|V_{n}\right|^{-1} \leq\left(1-|\beta|^{2}\right)^{-1}|\beta|^{n}$. This implies, for $s \geq 1$,

$$
\left|\Psi_{2 s}\right| \leq \sum_{n \geq 1}\left|V_{n}\right|^{-2 s} \leq\left(1-|\beta|^{2}\right)^{-2 s} \sum_{n \geq 1}|\beta|^{2 s n} \leq\left(1-|\beta|^{2}\right)^{-2 s-1}|\beta|^{2 s},
$$

and hence $\Psi_{2 s}(s \in \mathbb{N})$ are holomorphic for $|\beta|<1$. Set

$$
\Omega(\beta)=\left(8 \Psi_{2}+1\right) \chi(a)=12\left(3-128 \Psi_{2}^{3}-48 \Psi_{2}^{2}+320 \Psi_{6}\right) .
$$

Under the assumption $|\beta|<0.3$, we deduce from (54) that $\left|\Psi_{2}\right| \leq 1.1^{3}|\beta|^{2} \leq$ 0.12 , and that $\left|\Psi_{6}\right| \leq 1.1^{7}|\beta|^{6} \leq 0.002$. By these inequalities, for $|\beta|<0.3$ we get $|\Omega(\beta)| / 12 \geq 3-128\left|\Psi_{2}\right|^{3}-48\left|\Psi_{2}\right|^{2}-320\left|\Psi_{6}\right|>1$, and $\left|8 \Psi_{2}+1\right| \geq 1-$ $8\left|\Psi_{2}\right|>0.03$. Hence, in the domain $|\beta|<0.3$, the function $\sqrt{\chi(a)} \neq 0, \infty$ is holomorphic. Note that $\beta=\beta(a)$ conformally maps the domain $\mathbb{C} \backslash\{a=y i \mid$ $-2 \leq y \leq 2\}$ to the $\operatorname{disc}|\beta|<1$. To examine the corresponding domain around $a=\infty$, observe that for $|z|<1 / 7$,

$$
|\sqrt{1+z}-1| \leq\left|\int_{0}^{z} \frac{2}{3}(1+t)^{3 / 2} d t\right| \leq \frac{2}{3}\left(\frac{8}{7}\right)^{3 / 2}|z| \leq 0.8146|z| .
$$

Using this estimate, we have $|2 \beta|=\left|a\left(1-\sqrt{1+4 a^{-2}}\right)\right| \leq 0.8146\left|4 a^{-1}\right|=$ $3.2584|a|^{-1}$, provided that $\left|4 a^{-2}\right|<1 / 7$. This fact implies that the image of the domain $|a|>5.431$ is contained in the disc $|\beta(a)|<0.3$. By (54) and (55) we see

$$
\chi(a)=36+O\left(\beta^{2}\right)=36+O\left(a^{-2}\right) \quad \text { as } a \rightarrow \infty .
$$

To determine the branch of $\eta$, recall that

$$
\eta=(2 K / \pi)^{2}\left(1-2 k^{2}\right)=1-24 \Phi_{2}
$$

(cf. Section 4.4 and (29)). Since $|\alpha-\beta|\left|U_{n}\right| \geq|\beta|^{-n}\left(1-|\beta|^{2}\right)$, we have $\Phi_{2}=O\left(\beta^{2}\right)=O\left(a^{-2}\right)$, so that $\eta(a)=1+O\left(a^{-2}\right)$ as $a \rightarrow \infty$. This fact combined with (56) implies the assertion (i).

To determine the sign of $\eta(1)$, we use the following numerical values for $a=1$ :

$$
\Psi_{2}=1.2072919 \ldots, \quad \Psi_{6}=1.0016249 \ldots, \quad \Phi_{2}=0.485264 \ldots
$$

Then we have $\chi(1)=31.88115 \ldots=(5.6463 \ldots)^{2}$. On the other hand, $\eta(1)=-10.64633 \ldots$ by $(57)$. Consideration of these values leads to the sign - of the square root. Similarly, for $a=2$, using the values

$$
\Psi_{2}=0.2839243 \ldots, \quad \Psi_{6}=0.0156465 \ldots, \quad \Phi_{2}=0.1622974 \ldots,
$$


we get $\eta(2)=-2.89513 \ldots$ and $\chi(2)=(2.1048 \ldots)^{2}$, which yields the sign + in (53). Finally, we consider the case $a \geq 2.4$. Since $V_{2}=a^{2}+2=$ $a\left(a+2 a^{-1}\right) \geq 3.23 a$, we have $V_{n} \geq 3.23 a^{n-1}(n \geq 2)$, which yields $\Psi_{2}=$ $\sum_{n \geq 1} V_{n}^{-2} \leq a^{-2}+3.23^{-2} a^{-2} \sum_{j \geq 0} a^{-2 j}<1.116 a^{-2}<0.194$ for $a \geq 2.4$. Hence (55) implies $\chi(a)>1$ for $a \geq 2.4$. Combining this fact with (56) and $\eta(a)=1+O\left(a^{-2}\right)(a \rightarrow \infty)$, and taking the continuity of $\eta(a)$ into account, we deduce formula (53) for $a \geq 2.4$, which completes the proof.

5.2. Proof of Theorem 7. Since

$$
\left|\Psi_{2 s}^{*}\right| \leq \sum_{n \geq 1}\left|V_{n}\right|^{-2 s} \leq\left(1-|\beta|^{2}\right)^{-2 s-1}|\beta|^{2 s},
$$

we have $\left|\Psi_{2 s}^{*}\right| \leq 1.1^{2 s+1}|\beta|^{2 s}$ for $|\beta|<0.3$, so that

$$
\left|\chi_{2}(a)\right| \geq 16-64\left|\Psi_{2}^{*}\right|-9216\left|\Psi_{4}^{*}\right|^{2}-576\left|\Psi_{4}^{*}\right|-1920\left|\Psi_{6}^{*}\right|>0.7
$$

for $|\beta|<0.28$. This implies that $\sqrt{\chi_{2}(a)}$ is holomorphic for $|\beta|<0.28$. By the same argument as in Section 5.1, it is holomorphic for $|a|>5.819$. Recall the relation

$$
\theta=(2 K / \pi)^{2}=8 \Psi_{2}+1
$$

(cf. (44)). Observing the estimates $\Psi_{2}=O\left(a^{-2}\right), \chi_{1}(a)=-3+O\left(a^{-4}\right)$, and $\chi_{2}(a)=16+O\left(a^{-2}\right)$ as $a \rightarrow \infty$, we have $\theta(a)=\chi_{1}(a)+\sqrt{\chi_{2}(a)}$ for $|a|>5.819$, where the branch is taken so that $\sqrt{\chi_{2}(\infty)}=4$. In this way the assertion (i) is verified.

When $a=1$, by numerical computation we have

$$
\Psi_{2}^{*}=0.9370204 \ldots, \quad \Psi_{4}^{*}=0.9912040 \ldots, \quad \Psi_{6}^{*}=0.9988644 \ldots ;
$$

and relation (58) together with the numerical value of $\Psi_{2}$ (cf. Section 5.1) yields $\theta(1)=10.65833 \ldots$ Similarly when $a=2$,

$$
\Psi_{2}^{*}=0.2265861 \ldots, \quad \Psi_{4}^{*}=0.0617537 \ldots, \quad \Psi_{6}^{*}=0.0156036 \ldots,
$$

and $\theta(2)=3.27139 \ldots$ Using these numerical values, we can check the equalities for $a=1,2$. For $a \geq 2.5$, observe that $V_{n} \geq 3.3 a^{n-1}(n \geq 2)$. Then for $a \geq 2.5$ we have $\Psi_{6}^{*} \leq \sum_{m \geq 1} V_{2 m-1}^{-6} \leq a^{-6}+3.3^{-6} a^{-12} \sum_{j \geq 0} a^{-12 j} \leq$ $1.00001 a^{-6}<0.03 a^{-2}$ and $\Psi_{2}^{*} \geq V_{1}^{-2}-V_{2}^{-2} \geq\left(a^{2}+2\right)^{-1}>0.75 a^{-2}$, so that $\chi_{2}(a) \geq 7+64 \Psi_{2}^{*}-1920 \Psi_{6}^{*}>7-9.6 a^{-2}>5$, which implies the assertion.

5.3. Proof of Theorem 5. Put $\xi=-5 / 24+\lambda$. Then (3) is written in the form

$$
\lambda^{3}-3 \gamma_{1} \lambda-2 \gamma_{2}=0,
$$

where $\gamma_{1}$ and $\gamma_{2}$ are as given in the theorem, which may be regarded as functions of $a \in \mathbb{C}$ or $\beta$. Since $|\alpha-\beta|\left|U_{n}\right| \geq|\beta|^{-n}\left(1-|\beta|^{2}\right)$, we infer for $s \geq 1$ that $\left|\Phi_{2 s}^{*}\right| \leq\left(1-|\beta|^{2}\right)^{-2 s-1}|\beta|^{2 s}$. Hence $\Phi_{2 s}^{*}(s \in \mathbb{N})$ are holomorphic for $|\beta|<1$. In particular, for $|\beta|<0.2$, we have $\left|\Phi_{2}^{*}\right|<0.046,\left|\Phi_{4}^{*}\right|<0.002$ 
and $\left|\Phi_{6}^{*}\right|<0.0001$. Using (51), we obtain $\left|\gamma_{1}\right|>1.8,\left|\gamma_{2}\right|<2.3$ for $|\beta|<0.2$, and $\gamma_{1} \rightarrow 1129 \cdot 24^{-2}, \gamma_{2} \rightarrow-17963 \cdot 24^{-3}$ as $\beta \rightarrow 0($ or $a \rightarrow \infty)$.

To determine the branch of $\xi$, we note that $\xi(a)=\eta(a) / 4$ (cf. (57)), which implies $\xi \rightarrow 1 / 4$ as $a \rightarrow \infty$. Let us find the solution of (59) corresponding to $\xi$. Such a solution may be expressed in the form

$$
\lambda_{0}=\gamma_{1} / \varrho_{0}+\varrho_{0}, \quad \varrho_{0}=\sqrt[3]{\gamma_{2}+\sqrt{\gamma_{1}^{3}-\gamma_{2}^{2}} i}
$$

around $\beta=0$, where the branches should be chosen so that $24^{6}\left(\gamma_{1}^{3}-\gamma_{2}^{2}\right) \rightarrow$ $1129^{3}-17963^{2}>0, \xi \rightarrow 1 / 4$, namely, $\lambda_{0} \rightarrow 11 / 24$ as $\beta \rightarrow 0$. The second condition is satisfied if $(52)$ is valid. Furthermore, provided $|\beta|<0.2$, it is easy to see that $\left|\gamma_{1}^{3}-\gamma_{2}^{2}\right| \geq\left|\gamma_{1}^{3}\right|-\left|\gamma_{2}^{2}\right|>1.8^{3}-2.3^{2}>0$, and that $\gamma_{2}+\sqrt{\gamma_{1}^{3}-\gamma_{2}^{2}} i \neq 0$. Hence $\varrho_{0}(\beta)$ is holomorphic for $|\beta|<0.2$. By the same numerical argument as in Section 5.1, we can verify that the image of the domain $|a|>8.146$ is contained in the disc $|\beta|<0.2$. In this way the assertion (i) is proved.

If $a \geq 2.6$, then $\Phi_{2 s}^{*} \leq 10.76^{-s}$, because $(\alpha-\beta)^{2}=a^{2}+4 \geq 10.76$. Hence we have $\gamma_{1} \geq 1.44$ and $-1.68 \leq \gamma_{2} \leq 1.01$, implying $\gamma_{1}^{3}-\gamma_{2}^{2}>0.16$ for $a \geq 2.6$. This fact combined with the continuity of $\xi$ with respect to $a$ implies the assertion (ii).

When $a=1$, by numerical computation, we obtain

$$
\Phi_{2}^{*}=0.0335078 \ldots, \quad \Phi_{4}^{*}=0.0020616 \ldots, \quad \Phi_{6}^{*}=0.0001145 \ldots
$$

Then we have $\gamma_{1}(1)=1.836371 \ldots, \gamma_{2}(1)=-0.624754 \ldots$, and $\xi(1)=$ $\eta(1) / 4=-2.66158 \ldots$ (cf. Section 5.1). Similarly, when $a=2$, we have

$$
\Phi_{2}^{*}=0.0980088 \ldots, \quad \Phi_{4}^{*}=0.0146727 \ldots, \quad \Phi_{6}^{*}=0.0019227 \ldots,
$$

implying $\gamma_{1}(2)=1.079707 \ldots, \gamma_{2}(2)=0.766330 \ldots$, and $\xi(2)=\eta(2) / 4=$ $-0.72378 \ldots$. Using these numerical values, we can determine the branches as in the assertion (iii).

\section{References}

[1] M. Abramowitz and I. A. Stegun, Handbook of Mathematical Functions, Dover, New York, 1965.

[2] R. André-Jeannin, Irrationalité de la somme des inverses de certaines suites récurrentes, C. R. Acad. Sci. Paris Sér. I Math. 308 (1989), 539-541.

[3] R. Apéry, Irrationalité de $\zeta(2)$ et $\zeta(3)$, Astérisque 61 (1979), 11-13.

[4] F. Beukers, A note on the irrationality of $\zeta(2)$ and $\zeta(3)$, Bull. London Math. Soc. 11 (1979), 268-272.

[5] P. Bundschuh and K. Väänänen, Arithmetical investigations of a certain infinite product, Compos. Math. 91 (1994), 175-199.

[6] H. Cohen, Démonstration de l'irrationalité de $\zeta(3)$ (d'après R. Apéry), in: Séminaire de Théorie des Nombres, Grenoble, 1978-1979, VI.1-VI.9. 
[7] D. Duverney, Irrationalité de la somme des inverses de la suite de Fibonacci, Elem. Math. 52 (1997), 31-36.

[8] D. Duverney, Ke. Nishioka, Ku. Nishioka, and I. Shiokawa, Transcendence of Jacobi's theta series, Proc. Japan Acad. Ser. A Math. Sci. 72 (1996), 202-203.

[9] - , - - - - , Transcendence of Rogers-Ramanujan continued fraction and reciprocal sums of Fibonacci numbers, ibid. 73 (1997), 140-142.

[10] H. Hancock, Theory of Elliptic Functions, Dover, New York, 1958.

[11] A. Hurwitz und R. Courant, Vorlesungen über allgemeine Funktionentheorie und elliptische Funktionen, Springer, Berlin, 1925.

[12] C. G. J. Jacobi, Fundamenta Nova Theoriae Functionum Ellipticarum, Königsberg, 1829 .

[13] Th. Koshy, Fibonacci and Lucas Numbers with Applications, Wiley-Interscience, 2001.

[14] L. Navas, Analytic continuation of the Fibonacci Dirichlet series, Fibonacci Quart. 39 (2001), 409-418.

[15] Yu. V. Nesterenko, Some remarks on $\zeta(3)$, Mat. Zametki 59 (1996), 865-880, 960 (in Russian); English transl.: Math. Notes 59 (1996), 625-636.

[16] - Modular functions and transcendence questions, Mat. Sb. 187 (1996), 65-96 (in Russian); English transl.: Sb. Math. 187 (1996), 1319-1348.

[17] S. Ramanujan, On certain arithmetical functions, Trans. Cambridge Philos. Soc. 22 (1916), 159-184.

[18] E. T. Whittaker and G. N. Watson, Modern Analysis, 4th ed., Cambridge Univ. Press, Cambridge, 1927.

[19] I. J. Zucker, The summation of series of hyperbolic functions, SIAM J. Math. Anal. 10 (1979), 192-206.

[20] V. V. Zudilin, One of the numbers $\zeta(5), \zeta(7), \zeta(9), \zeta(11)$ is irrational, Uspekhi Mat. Nauk 56 (2001), no. 4, 149-150 (in Russian); English transl.: Russian Math. Surveys 56 (2001), 774-776.

Fachhochschule für die Wirtschaft

University of Applied Sciences

Freundallee 15

D-30173 Hannover, Germany

E-mail: carsten.elsner@fhdw.de
Department of Mathematics Keio University 3-14-1 Hiyoshi, Kohoku-ku Yokohama, 223-8522 Japan E-mail: shimomur@math.keio.ac.jp shiokawa@beige.ocn.ne.jp 